\title{
An examination of maternal contributors and potential modifiers of fetal growth in pregnancy
}

Zachary M. Ferraro

\begin{abstract}
A greater understanding of critical periods of body weight regulation, including pregnancy, may aid in efforts to optimize weight management strategies for the mother and her baby. The gestational period has been implicated to play, in the child, a vital role in the developmental origins of obesity and other cardiometabolic diseases later in life. Therefore, we initially examined existing literature on the role of maternal obesity and its link to pediatric obesity and documented the known underlying physiological mechanisms responsible for this relationship, while suggesting potential intervention targets that may improve maternal-fetal outcomes. In a second paper, we aimed to quantify maternal predictors of large for gestational-age neonates in the Ottawa and Kingston birth cohort with specific hypotheses verifying the independent contribution of maternal prepregnancy body mass index (BMI) and excessive gestational weight gain (GWG) to fetal overgrowth. This paper also highlighted the clinical utility of the revised 2009 Institute of Medicine GWG guidelines and discussed the potential role of physiological factors underlying the observed associations. As follow-ups to our population-level analysis, papers 3 and 4 highlighted how the insulin-like growth factor (IGF) axis, a vital regulator of growth and development, may be compromised at the molecular level in cases of maternal obesity (paper 3) and excessive GWG (paper 4). In paper 3, we showed that maternal obesity is associated with attenuated expression of IGF binding protein-4 (IGFBP-4) in umbilical cord blood and discussed how this may preferentially promote fetal adipogenesis. The effects of excessive GWG on IGF axis protein expression were addressed in paper 4, where we showed that independent of prepregnancy BMI, excessive weight gain during pregnancy is associated with increased expression of IGFBP-3 in maternal circulation in normoglycemic term pregnancies. In that paper we discussed the potential inhibitory role of IGFBP-3 on adipogenesis and how it relates to glucose intolerance during pregnancy. Recognizing that both obesity and excessive GWG can alter physiological processes in a mother and her baby, we concluded that appropriate evidence-based interventions are warranted to best optimize outcomes. In paper 5, we discussed the results of a study that sought to assess patient information channels and knowledge of nutrition and physical activity during pregnancy. The intent was that these findings be applied to best-design efficacious strategies that cater to the needs of our target group of pregnant women. In our analysis we showed that the majority of pregnant women studied would be willing to participate in a lifestyle intervention for their own personal health and that of their child. Of great interest was the observation that most women were not informed of the importance of pregnancy-specific energy intake, or made aware of their own healthy GWG targets. Additionally, many of the respondents reported that they did not receive information that pertained to appropriate physical activity recommendations, even though the vast majority of participants considered this lifestyle modality to be safe during their pregnancy. Finally, in paper 6 we built on the results of our previous work and evaluated the risks and benefits of physical activity during pregnancy on maternal-fetal outcomes through a review of the literature and noted that engaging in nonsedentary pursuits during gestation may aid in maternal weight regulation, protect against metabolic disorders, and optimize neonatal birth weight and body composition. Overall, the collective nature of the papers presented in this dissertation provides qualitative and quantitative evidence to support not only the complexity of body weight regulation in the mother and her baby, but also highlights potential avenues for intervention that may improve maternal-fetal outcomes during this critical period.
\end{abstract}

Received 5 November 2012. Accepted 9 November 2012.

Z.M. Ferraro., ${ }^{\dagger}$ HALO Research Group, Children's Hospital of Eastern Ontario Research Institute, 401 Smyth Road, Ottawa, ON K1H 8L1, Canada.

E-mail for correspondence: zach.ferraro@gmail.com.

*Advisors: Dr. Kristi B. Adamo, MSc, PhD (primary advisor), Research Scientist, Healthy Active Living and Obesity (HALO) Research Group, Children's Hospital of Eastern Ontario Research Institute; Dr. Denis Prud'homme, MD, MSc (co-advisor), Full Professor, School of Human Kinetics, Faculty of Health Sciences, University of Ottawa.

${ }^{\dagger}$ Dr. Ferraro is currently working as a part-time clinical research associate in the Adamo lab at the HALO research group. He is a Certified Exercise Physiologist (CSEP-CEP) and continues to be involved with Dr. Adamo's Maternal Obesity Management (MOM) trial. His research interests include obesity, gestational weight gain, and cardiometabolic risk factors and how these influence placenta nutrient transport and fetal growth. 